\title{
Positive and negative successive contrast effects following multiple shifts in reward magnitude under high drive and immediate reinforcement
}

\author{
FREDERICK R. MAXWELL \\ Southwest Missouri State University, Springfield, Missouri 65802 \\ and \\ RICHARD S. CALEF, D. W. MURRAY, J. C. SHEPARD, and R. A. NORVILLE \\ West Virginia Wesleyan College, Buckhannon, West Virginia 26201
}

\begin{abstract}
Maltiple shifts in reward amount were given to rats in a straight runway. Both positive and negative contrast effects were observed. A positive contrast effect was observed, however, only if the shift from small to large reward occurred while the subject was evidencing a negative contrast effect. Implications for current conceptions of reinforcement contrast are discussed.
\end{abstract}

The research in instrumental conditioning is replete with data showing the effect of shifts in reward magnitude (Black, 1968). Specifically, many studies (e.g., Capaldi \& Lynch, 1967; Crespi, 1942; Ehrenfreund, 1971; Gonzales, Gleitman, \& Bitterman, 1962) have shown that subjects shifted from large (L) to small (S) magnitude of reward run more slowly than subjects maintained on small magnitude of reward, a phenomenon designated as the "depression effect" or the negative contrast effect (NCE). Previous literature (Black, 1968; Dunham, 1968) has also reported that under high drive a shift from small to large magnitude of immediate reward does not produce greater speeds than does consistent large magnitude of immediate reward. Recently, however, two procedures have emerged which seem to produce a reliable "elation" effect, or positive contrast effect (PCE). The first procedure simply involves superimposing delay of reward on the standard magnitude shifts described above (Mellgren, 1972; Shanab, Sanders, \& Premack, 1969). According to Mellgren (1972), previous failure to obtain a PCE may have been due to the fact that rats in the large-reward control group were running at or near their physiological limit. Delay of reward, then, by reducing overall running speed prevents this ceiling effect. The second procedure exposes the subject to a decrease in reward magnitude prior to the subsequent increase in reward magnitude, i.e., LSL training in which subjects are initially trained on large reward, shifted to small reward, and subsequently shifted back to large reward (Benefield, Oscos, \& Ehrenfreund, 1974). However, Capaldi and Lynch (1967) failed to obtain a PCE using this LSL procedure. While there are several procedural differences between the experiments of Benefield et al. and
Capaldi and Lynch, one difference that may have contributed to the conflicting results was the timing of the shift from small to large reward. Capaldi and Lynch shifted their subjects to large magnitude of reward after 15 trials of small magnitude of reward, while Benefield et al. shifted their subjects after only 5 trials on small magnitude of reward. More importantly perhaps, Capaldi and Lynch shifted their subjects after the NCE had dissipated, while Benefield et al. may have shifted their subjects during the NCE. Unfortunately, Benefield et al. did not include a small-reward control group to determine whether, at the point of the shift from small to large reward, their rats were showing a depression effect.

The primary purpose of the present study was to determine whether, under experimental procedures like those of Capaldi and Lynch and Benefield et al., i.e., LSL training, shifting from $S$ to $L$ while $S$ speeds are depressed below those of a small-reward control is prerequisite to obtaining a PCE (elation) under conditions of high drive and immediate reward. Four groups were used: a large-reward control (LLL), a small-reward control (SSS), and two experimental groups $\left(\mathrm{LS}_{\mathrm{d}} \mathrm{L}\right.$ and $\left.L S_{a} \mathrm{~L}\right)$. The first of these latter two groups was shifted from $S$ to $L$ during the Phase 2 $\mathrm{NCE}$, while the second was shifted from $S$ to $\mathrm{L}$ after the Phase 2 NCE had dissipated. We expected that only the former group would show a PCE.

A fourth phase for the two experimental groups and the small-reward control was included. Both experimental groups were shifted back to S, e.g., LS $_{d} L S$ and $L S_{a} L S$, while Group SSS was maintained on S, e.g., SSSS. Since the two experimental groups received different amounts of $S$ training in Phase 2 and performed differently in Phase 3, we thought this might lead to differences in Phase 4 performance. 
Specifically, the experimental group receiving the greater amount of Phase 2 training $\left(\mathrm{LS}_{\mathrm{a}} \mathrm{LS}\right)$ might be expected to show little depression in Phase 4 due to transfer from Phase 2 training (cf. Capaldi, 1972).

\section{METHOD}

\section{Subjects}

The subjects were 36 experimentally naive male rats, approximately 180 days old at the beginning of the experiment. Twelve rats were hooded and 24 were albino, all bred at West Virginia Wesleyan College.

\section{Apparatus}

The runway apparatus was essentially the same as that used by Ludvigson and Gay (1966), except that only one of the multiple parallel alleys was used. The alley comprised a $33.02-\mathrm{cm}$ white startbox, a $66.04-\mathrm{cm}$ white run section, and a $30.48-\mathrm{cm}$ white goalbox. The inner width and height of each section of the runway was $7.62 \mathrm{~cm}$. Photocell and clock circuitry provided independent measures of traversal times over the first $20.32-\mathrm{cm}$ (start time) and second $30.48-\mathrm{cm}$ (run time) segments of the run section. The apparatus also contained a solenoid-operated, opaque, guillotinetype, retrace door separating goal from alley section and an opaque Plexiglas ceiling.

\section{Procedure}

Fourteen days prior to the first day of training (Day 15), all rats were placed on a 23-h food-deprivation schedule, which was maintained throughout the study. On Days 7-14, the subjects were taken from the individual home cage and handled for about 2 min each. On Days 13 and 14, the subjects were placed in individual carrying cages and taken to the experimental room, where they were allowed to explore start and run sections of the apparatus while clocks, photocells, and doors were operated. On those days, approximately $1 \mathrm{~g}$ of $45-\mathrm{mg}$ Noyes pellets, identical to the subsequent reinforcement pellets, were incorporated into the subjects' daily food ration.

Three hoods and six albinos were assigned randomly to each of four groups. Group $\mathrm{LS}_{\mathrm{d}} \mathrm{LS}$ received 25 trials of large reward (12 pellets) during Phase 1 . During Phase 2, the rats received 10 trials of small reward (1 pellet) and were then shifted to large reward again (Phase 3). Following 15 trials in Phase 3, the subjects were shifted back to small reward for 20 trials (Phase 4). Group LS $\mathrm{S}_{\mathrm{a}} \mathrm{LS}$ subjects were treated identically, except they received 20 trials on small reward in Phase 2, 25 trials on large reward in Phase 3, and 10 trials on small reward in Phase 4. Group SSSS received consistent small reward for a total of 80 trials during the four phases of training. Group LLLL received consistent large reward for a total of $\mathbf{8 0}$ trials during the four phases of training. All groups received 1 trial on the first training day, 2 trials on the second day, 3 trials on the third day, 4 trials on the fourth day, and 5 trials on every day thereafter. Trials were administered to three squads of 12 subjects each, consisting of three rats from each group. The running order of subjects within a squad was randomized from day to day. The intertrial interval was approximately $7 \mathrm{~min}$, since a rat did not receive its second daily trial until all the subjects in a squad had completed their first trial. A running trial was initiated by placing a subject in the startbox. After a 3-sec orientation period, the start door was opened and the subject was allowed to traverse the runway. Following the subject's entry into the goalbox, the retrace door was dropped. The subject was allowed to consume the reward pellets and was then removed from the apparatus to a nearby holding cage to await his next trial.

Start and run latencies were converted to reciprocals, yielding start and run speeds, respectively.

\section{RESULTS}

Start and run speed data were essentially similar, but differences were generally larger and more reliable in the run measure. Thus, only run speeds are presented below.

\section{Phase 1: Acquisition}

As may be seen in Figure 1, during the first 7 days of training the high-reward groups (Groups LLLL, $\mathrm{LS}_{\mathrm{d}} \mathrm{LS}$, and $\mathrm{LS}_{\mathrm{a}} \mathrm{LS}$ ) developed faster run speeds than did the small-reward group (Group SSSS). A one-way analysis of variance performed on the data from Day 7 of training yielded a significant Groups effect $(F=3.70, d f=3 / 36, p<.05)$. Subsequent paired comparisons using the Neuman-Keuls procedure (all subsequent paired comparisons use this procedure) indicated that each of the large-reward groups ran reliably faster than the small-reward

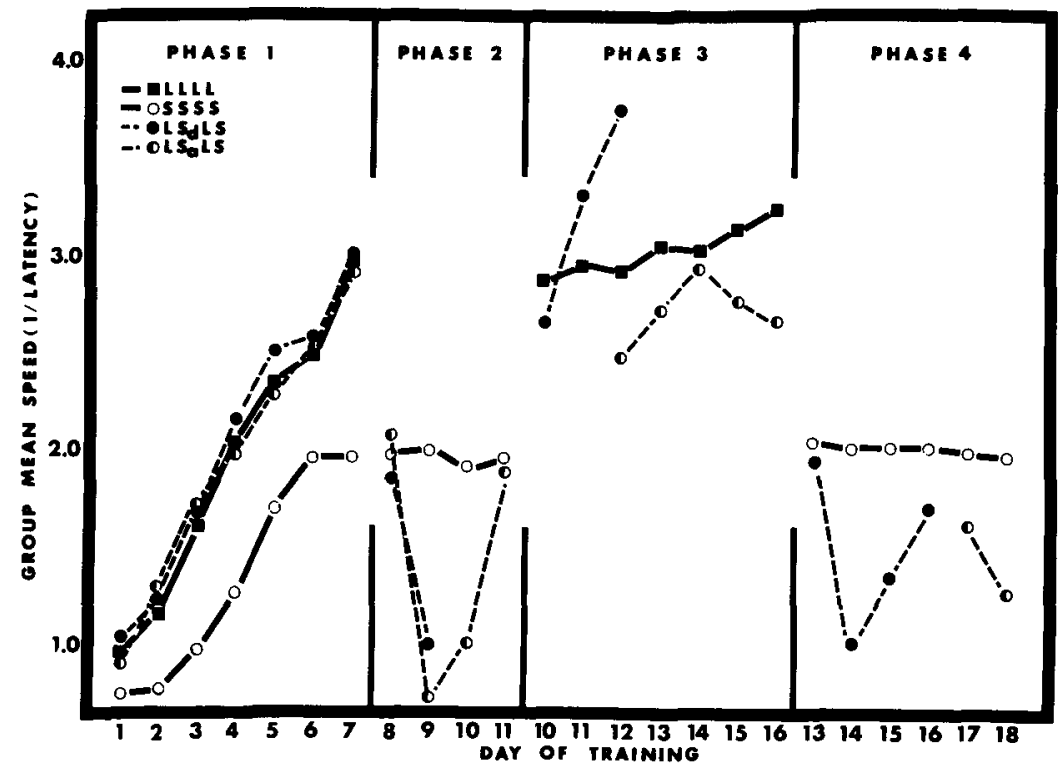

Figure 1. Group mean speed for each phase of the experiment as a function of day of training. Note that only the data for groups of interest are included in each phase of the experiment and that a given day's data may appear in different phases for different groups. 
group $(\mathrm{p}<.05)$ but did not differ reliably among themselves $(p>.05)$.

\section{Phase 2: Depression}

Looking at Phase 2 data in Figure 1, it is clear that both shift groups (e.g., $\mathrm{LD}_{\mathrm{d}} \mathrm{LS}$ and $\mathrm{LS}_{\mathrm{a}} \mathrm{LS}$ ) evidenced a depression of speeds relative to the smallreward control (Group SSSS). A one-way analysis of variance performed on the data from Day 9 yielded a significant Groups effect $(F=17.97$, df $=3 / 36, \quad p<.05$ ), and subsequent paired comparisons indicated that Groups LS $_{d} L S$ and LS $_{\mathrm{a}} \mathrm{LS}$ ran reliably slower than Group SSSS $(p<.05)$. The data from Group LLLL, although not shown in Figure 1, was included in this analysis. Paired comparisons indicated that Group LLLL ran reliably faster than each of the other three groups $(p<.05)$. Turning to the last day of Phase 2 (Day 11), it can be seen (Figure 1) that the depression of running speeds in Group LS $_{\mathrm{a}} \mathrm{LS}$ has dissipated. A separate one-way analysis performed on the data from Day 11 yielded a significant Groups effect $(F=4.64$, df $=3 / 36, p<.05)$. Although all groups were included in the analysis, only Groups SSSS and LS $_{\mathrm{a}} \mathrm{LS}$ are of interest with respect to Phase 2 performance. A paired comparison indicated that Group SSSS did not differ reliably from Group $\mathrm{LS}_{\mathrm{a}} \mathrm{LS}$ $(\mathrm{p}>.05)$.

\section{Phase 3: Elation}

The Phase 3 data were the data of major interest in this study. The Phase 3 data presented in Figure 1 suggest that Group LS $_{\mathrm{d}} \mathrm{LS}$ displayed faster running speeds than Group LLLL on Days 11 and 12. Oneway analysis of variance (including all groups) was performed on the data from Days 11 (same analysis mentioned in Phase 2 section) and $12(\mathrm{~F}=4.64$, $\mathrm{df}=3 / 36, \mathrm{p}<.05$, and $\mathrm{F}=8.42$, df $=3 / 36$, $\mathrm{p}<.05$, respectively). Subsequent paired comparisons indicated that on Day 11 Group $\mathrm{LS}_{\mathrm{d}} \mathrm{LS}$ did not differ reliably from Group LLLL $(p>.05)$, but that on Day 12 Group LS $_{d} L S$ did respond reliably faster than Group LLLL $(p<.05)$.

Phase 3 data for Group LS $_{\mathrm{a}}$ LS present a markedly different picture from that of Group LS $_{d} L S$. While the performance level of Group $\mathrm{LS}_{\mathrm{a}} \mathrm{LS}$ was enhanced relative to that of Group SSSS, inspection of Figure 1 indicates that at no time did the performance of Group LS $_{a} L S$ exceed that of Group LLLL. Analyses identical in nature of those mentioned previously and subsequent paired comparisons indicated that Group $\mathrm{LS}_{\mathrm{a}} \mathrm{LS}$ did not differ reliably from Group LLLL on Days 12, 14, or $16(\mathrm{p}>.05)$.

\section{Phase 4: Second Depression}

As may be seen in Figure 1, both Group ${ }^{2} S_{d} L S$ and Group LS $_{\mathrm{a}} \mathrm{LS}$ showed depressed running speed relative to Group SSSS in Phase 4. One-way analyses of variance performed on the data from Days 14 and 18 yielded significant Groups effects $(F=23.77$, df $=3 / 36, p<.05$, and $F=5.58$, df $=1 / 16$, $\mathrm{p}<.05$, respectively). Paired comparisons confirmed that on Day 14 Group $\mathrm{LS}_{\mathrm{d}} \mathrm{LS}$ ran reliably slower than did Group SSSS $(p<.05)$, while on Day 18 Group LS $_{\mathrm{a}} \mathrm{LS}$ ran reliably slower than did Group SSSS $(\mathrm{p}<.05)$.

\section{DISCUSSION}

The present results show quite clearly that whether or not an increase in reward amount, subsequent to a downward shift in reward amount, produces positive contrast is a function of the amount of Phase 2 training. Group $\mathrm{LS}_{\mathrm{d}} \mathrm{LS}$, which was shifted from small to large reward relatively early in Phase 2, evidenced a PCE in Phase 3. But Group $L^{2} \mathrm{LS}$, shifted much later in Phase 2, showed no PCE in Phase 3. Both experimental groups showed substantial NCEs in Phases 2 and 4.

In our view, the present experiment indicates a PCE only if performance is depressed at the time of the upshift in reward amount. Taken with those of previous investigations showing PCEs under conditions of delay (Mellgren, 1972; Shanab, Sanders, \& Premack, 1969) and punishment (Shanab \& White, 1972), our data suggest that the presence of an adverse state may be prerequisite to the occurrence of a PCE. This is similar to the suggestion by Benefield et al. (1974) that PCEs following delay of reward may be due to delay-engendered frustration. However, we noted previously that their study did not offer direct support for such a hypothesis.

Perhaps an increase in amount of reward does not by itself increase motivational level. While there is massive evidence supporting the motivational consequences of reward reduction (e.g., Amsel, $1958,1962)$, there is only one study showing motivational consequences of an upshift in amount of reward. Meyer and McHose (1968), using a double alley paradigm [introduced in the now well-known study of Amsel and Roussel (1952)], demonstrated that rats that received varied reward in the first alley ran faster in the second alley following the larger of the first-alley rewards than did control subjects receiving only large reward in the first alley. They termed this increase in speeds following large reward in the context of small reward an "elation effect." Note, however, that their design involves both upshifts and downshifts in reward amount, e.g., SL and LS. In the light of the present experiment, their "elation" effect may have been the result of the carryover of the effects of reward reductions rather than an effect of reward increases per se.

Our results also indicate that a prior history that 
includes depressed responding is not sufficient to insure positive contrast during a subsequent increase in reward amount. Likewise, prior commerce with the larger reward amount was not sufficient to produce subsequent positive contrast. Both of these conditions have been implicated in earlier research as possible sources of the PCE (Benefield, Oscos, \& Ehrenfreund, 1974; Calef, 1972). Both were present in Group $\mathrm{LS}_{\mathrm{a}} \mathrm{LS}$, yet no positive contrast was observed. Further, the observation that PCEs can be demonstrated reliably under conditions of high drive and immediate reward argues convincingly against an explanation of delay-engendered PCEs which appeal strictly to lowered response speeds, e.g., the ceiling effect hypothesis (Mellgren, 1972).

Our results, then, would seem to provide a possible explanation of the apparently contradictory results obtained by Capaldi and Lynch (1967) and Benefield et al. (1974). As we have already observed in the former study, Group LSLS was shifted from small reward (Phase 2) to large reward (Phase 3) after depression had apparently dissipated and, as in the present experimental Group $\mathrm{LS}_{\mathrm{a}} \mathrm{LS}$, no positive contrast was observed. Benefield et al., however, employing an essentially similar experimental design, shifted from small to large reward while the subjects were presumably (no small-reward control was included in their study) still evidencing depression and, as in the present experimental Group $L S_{d} L S$, they observed a reliable PCE.

The Capaldi and Lynch study differs from both Benefield et al. and the present study in at least one other respect. Capaldi and Lynch employed a spaced trial procedure (i.e., 24-h ITI), while in both Benefield et al. and our study much shorter ITIs $(21 / 2 \mathrm{~h}$ and $10 \mathrm{~min}$, respectively) were used. Capaldi (1972) has shown that ITI is an important determinant of the NCE. Specifically, his investigations indicate that NCEs are larger, and presumably more reliable, under massed-trial than under spaced-trial conditions. Thus it is possible that the spaced-trial procedures employed by Capaldi and Lynch contributed to their failure to observe a PCE. However, an ITI-based explanation of these discrepancies in experimental findings cannot adequately account for the present data, because in Phase 3 both the presence and absence of a PCE was observed in the absence of any differences in ITI.

In Phase 4 of the present experiment, the observation of reliable NCEs for both experimental groups is testimony to the hardiness of the depression phenomenon. These results also seem to be in conflict with those of Capaldi and Lynch. Specifically, using a similar design, i.e., LSLS, they observed no Phase 4 NCE. However, as noted above, they employed a spaced-trial procedure, and, as Capaldi (1972) has shown, this procedure leads to marked reductions in the NCE during such "transfer shifts."
The absence of marked differences in the Phase 4 NCE for the experimental groups did not support our hypothesis of differential transfer effects from Phase 2 to Phase 4.

Finally, certain aspects of the data do not support an unmodified "reinforcement level principle" as proposed by Capaldi (1974). Briefly, Capaldi proposes that "if obtained reward is greater than expected reward, the available stimuli will acquire a greater capacity than otherwise to elicit the reaction." Further, "Adjustment of expected reward to prevailing reward conditions will be fastest where no specific former expected reward level has been established .... and it will be slower the stronger is the former expected reward." In short, Capaldi views the $P C E$ as resulting from a greater than normal conditioning effect of large reward following small reward. Further, the size of the PCE should increase (up to some maximum) with increasing amounts of prior small-reward training. Our results are clearly inconsistent with such a prediction. According to Capaldi's analysis, Group $\mathrm{LS}_{\mathrm{a}} \mathrm{LS}$, which received a much greater amount of Phase 2 smallreward training than Group $\mathrm{LS}_{\mathrm{d}} \mathrm{LS}$, should have developed the larger PCE. In fact, Group $\mathrm{LS}_{\mathrm{a}} \mathrm{LS}$ did not develop a PCE, while Group $\mathrm{LS}_{\mathrm{d}} \mathrm{LS}$ clearly did show a PCE.

Our results suggest that the PCE be viewed as a contextual reinforcement phenomenon having as a prerequisite the presence of some type of negative affect state. Such a view would not be incompatible with major theoretical formulations dealing with the reward contrast literature (Amsel, 1958, 1967; Capaldi 1972, 1974). Indeed, Surridge, Boehnert, and Amsel (1966) have suggested specifically that increases in performance in similar circumstances might be due to an added conditioned frustrative drive $\left(\mathrm{r}_{\mathrm{F}}\right)$. Our view is that primary frustration or any aversive emotional state may temporarily have the same effect. In a recent article that came to our attention after the present paper was substantially completed, McCain and Cooney (1975) suggest an approach that combines Capaldi's reinforcement level principle with an arousal concept. In brief, their view is that prior negative arousal may provide a greater positive contrast, thus they attribute the PCE to a greater positive arousal rather than to a carryover of negative arousal as suggested above. The difference seems one of theoretical convenience rather than necessity. On either approach, those variables affecting the magnitude, timing, duration, etc., of negative emotional states should be variables affecting the PCE.

\section{REFERENCES}

AMSEL. A. The role of frustrative nonreward in noncontinuous reward. Psychological Bulletin, 1958, 55, 102-119. 
Amsel, A. Frustrative nonreward in partial reinforcement and discrimination learning: Some recent history and theoretical extensions. Psychological Review, 1962, 69, 306-328.

AMSEL, A. Partial reinforcement effects on vigor and persistence: Advances in frustration theory derived from a variety of within subject experiments. In K. W. Spence \& J. T. Spence (Eds.), The psychology of learning and motivation (Vol. 1). New York: Academic Press, 1967. Pp. 1-65.

AMSEl. A., \& Roussel, J. Motivational properties of frustration: I. Effect on a running response of the addition of frustration to the motivational complex. Journal of Experimental Psychology, 1952, 43, 363-368.

Benefield, R., Oscos, A., \& Ehrenfreund, D. Role of frustration in successive positive contrast. Joumal of Comparative and Physiological Psychology, 1974, 86, 648-651.

BLACK, R. W. Shifts in magnitude of reward and contrast effects in instrumental and selective learning: A reinterpretation. Psychological Review, 1968, 75, 114-126.

CaLEF, R. C. The effect of large and small magnitude of intertrial reinforcement on successive contrast effects. Psychonomic Science, 1972, 29, 309-312.

CAPAldi, E. J. Successive negative contrast effect: Intertrial interval, type of shift, and four sources of generalization decrement. Journal of Experimental Psychology, 1972, 98, 433-438.

CAPAldi, E. J. Partial reward either following or preceeding consistant reward: A case of reinforcement level. Journal of Experimental Psychology, 1974, 102, 954-962.

CAPAldi, E. J., \& LynCh, D. Repeated shifts in reward magnitude: Evidence in favor of an associational and absolute (non contextual) interpretation. Joumal of Experimental Psychology, 1967, 75, 226-235.

Crespi, L. P. Quantitative variations in incentive and performance in the white rat. American Journal of Psychology, 1942, 55, 467-517.
Dunham, P. J. Contrasted conditions of reinforcement: A selective critique. Psychological Bulletin, 1968, 69, 295.315.

EHRENFREUND, D. The effect of drive on successive magnitude shifts in rats. Journal of Comparative and Physiological Psychologv, 1971, 76, 418-423.

Gonzales, R. C., Gleitman, H., \& Bitrerman, M. E. Some observations on the depression effect. Journal of Comparative and Physiological Psychology, 1962, 55, 578-581.

Ludvigson, H. W., \& GAY, S. E. Differential reward conditioning: $\mathrm{S}-$ contrast as a function of the magnitude of $\mathrm{S}+$. Psychonomic Science, 1966, 5, 289-290.

MCCAIN, G., \& CooneY, J. PCE: I. The effects of three reward magnitude shifts. Bulletin of the Psychonomic Society, 1975, 6 , 523-526.

Mellgren, R. L. Positive and negative contrast effects using delayed reinforcement. Learning and Motivation, 1972, 3. 185-193.

MEYer, P. A., \& McHose, J. H. Facilitative effects of reward increase: An apparent "elation effect". Psychonomic Science, $1968,13,165-166$.

Shanab, M. E., Sanders, R., \& Premack, D. Positive contrast in the runway obtained with delay of reward. Science, 1969, 164, 724-725.

Shanab, M. E., \& Whte, R. Positive contrast obtained with punishment. Journal of General Psychology, 1972, 86, 247-251.

SurRidge, C. T., Boehnert, J., \& AMSEL, A. The effect of interpolated extinction on the reacquisition of partially and continuously rewarded responses. Journal of Experimental Psychology, 1966, 72, 564-570.

(Received for publication February 9, 1976; revision accepted August 16.1976.) 